\title{
Power Scattering and Absorption Mediated by Cloak/Anti-Cloak Interactions: A Transformation-Optics Route towards Invisible Sensors
}

\author{
Giuseppe Castaldi, ${ }^{1}$ Ilaria Gallina, ${ }^{1}$ Vincenzo Galdi, ${ }^{1, *}$ Andrea Alù, ${ }^{2}$ and Nader \\ Engheta $^{3}$ \\ ${ }^{1}$ Waves Group, Department of Engineering, University of Sannio, Corso Garibaldi 10\%, \\ I-82100 Benevento, Italy \\ 2 Department of Electrical and Computer Engineering, The University of Texas at Austin, \\ Austin, TX 78712, USA \\ 3 Department of Electrical and Systems Engineering, University of Pennsylvania, \\ Philadelphia, PA 19104, USA \\ *Corresponding author: vgaldi@unisannio.it
}

\begin{abstract}
The suggestive idea of "cloaking" an electromagnetic sensor, i.e., strongly reducing its visibility (scattering) while maintaining its field-sensing (absorption) capabilities, has recently been proposed in the literature, based on scattering-cancellation, Fano-resonance, or transformation-optics approaches. In this paper, we explore an alternative, transformation-optics-based route, which relies on the recently-introduced concept of "anti-cloaking." More specifically, our proposed approach relies on a suitable tailoring of the competing cloaking and anti-cloaking mechanisms, interacting in a twodimensional cylindrical scenario. Via analytical and parametric studies, we illustrate the underlying phenomenology, identify the critical design parameters, and address the relevant optimality and tradeoff issues, taking also into account the effect of material losses. Our results confirm the envisaged potentials of the proposed transformation-optics approach as an attractive alternative route to sensor cloaking.
\end{abstract}

OCIS codes: (230.3205) Invisibility cloaks; (230.0230) Optical devices; (160.1190) Anisotropic optical materials; (260.2710) Inhomogeneous optical media; (260.2110) Electromagnetic optics. 


\section{Introduction}

During the past few years, there has been a growing interest in the development of strategies and devices to achieve invisibility of objects to waves of various nature (electromagnetic, acoustic, elastic, matter). With special reference to the electromagnetic (EM) case, among the most prominent approaches to passive "invisibility cloaking," it is worth mentioning those based on scattering cancellation [1,2], anomalous localized resonances [3], transformation optics (TO) [4,5], inverse design of scattering optical elements [6], transmission-line networks [7], and tapered waveguides [8]. The reader is also referred to [9-12] for related experimental studies at microwave and optical frequencies.

In the TO approach to cloaking, of particular interest for what follows, the rerouting of the impinging energy around the concealed object is first designed in a fictitious curvedcoordinate space containing a "hole," and it is subsequently translated into a conventionally flat, Cartesian space, filled by an anisotropic and spatially inhomogeneous "transformation medium" which embeds the coordinate-mapping effects $[4,5,13,14]$. Starting from this basic concept, a variety of extensions and twists have been proposed, including the carpet cloak [15-20], open cloak [21], cloak at a distance [22], anti-cloak [23,24], superscatterer [25] and superabsorber [26], invisible gateway [27], as well as the broad framework of "illusion optics" [28].

In connection with the anti-cloaking concept, directly related to the subject of this paper, it was shown in [23] that, in spite of the generally assumed impenetrability of an ideal cylindrical cloak shell [29-31] (implying a lossless, anisotropic, spatially inhomogeneous medium, with extreme values of the constitutive parameters ranging from zero to infinity), field penetration in the cloaked region may actually occur in the presence of a suitably designed complementary double-negative (DNG) transformation cylindrical shell of "undoing" (partially or completely) the cloak transformation. Expanding upon this concept, in [24], we studied a more general scenario featuring a cloak and an anti-cloak separated by a vacuum layer, and showed that: i) anti-cloaking-type effects and interesting field tunneling phenomena may also be achieved with double-positive (DPS) or single-negative (SNG) transformation media, thereby relaxing some of the practical feasibility limitations; ii) besides the originally proposed "cloaking countermeasure" application in [23], alternative application scenarios may be envisaged for which a (multiply-connected) region of space may be cloaked, while maintaining the capability of somehow "sensing" the outside field from the inside. A similar idea, within the suggestive framework of "sensor cloaking," was recently explored in [32] (via a scattering-cancellation approach), in [33] (via a Fano-resonance-type approach), and in [34] (via a TO-based approach).

In this paper, we further elaborate on the above concepts, exploring more in detail the possibility to apply them to sensor-cloaking. In particular, for the same two-dimensional 
(2-D) scenario considered in [24], consisting of a metamaterial cylindrical target surrounded by a cloak and an anti-cloak separated by a vacuum gap, we present some analytical results and parametric studies aimed at gaining a deeper insight in the tradeoff between power absorption and scattering for a slightly lossy target (mimicking the sensor loading effects). In this framework, we show that the cloak/anti-cloak interactions can be tailored so as to allow sensible power absorption by the target, while still guaranteeing very weak overall scattering, thereby indicating an alternative TO-based route to sensor cloaking.

Accordingly, the rest of the paper is organized as follows. In Sec. 2, we introduce the problem geometry and formulation. In Sec. 3, we outline the general analytical solution and the relevant derivations. In Sec. 4, we illustrate and discuss some representative results from a comprehensive parametric study, and, in Sec. 5, we address some implementation-related issues. Finally, in Sec. 6, we provide some brief concluding remarks and hints for future research.

\section{Problem geometry and statement}

The scenario of interest is the same as the one considered in [24] and, as illustrated in Fig. 1 , entails a four-layer cylindrical configuration of radii $R_{\nu}, \nu=1, \ldots, 4$ in the physical space $(x, y, z)$. More specifically, such configuration features an innermost metamaterial cylindrical target of radius $R_{1}$ (with electric permittivity $\varepsilon_{1}$ and magnetic permeability $\mu_{1}$ ) surrounded by two transformation-medium layers separated by a vacuum gap, and is obtained via a piecewise-linear radial coordinate mapping,

$$
r^{\prime}=f(r)= \begin{cases}r, \quad r<R_{1}, \quad r>R_{4}, \\ R_{1}\left(\frac{R_{2}+\Delta_{2}-r}{R_{2}+\Delta_{2}-R_{1}}\right), & R_{1}<r<R_{2}, \\ R_{4}\left(\frac{r-R_{3}+\Delta_{3}}{R_{4}-R_{3}+\Delta_{3}}\right), & R_{3}<r<R_{4},\end{cases}
$$

from an auxiliary fictitious space $\left(x^{\prime}, y^{\prime}, z^{\prime}\right)$ featuring a homogeneous circular cylinder with constitutive parameters $\varepsilon_{1}$ and $\mu_{1}$, and radius $R_{2}>R_{1}$. The analytical expressions of the constitutive tensors characterizing the cloak and anti-cloak transformation media are systematically derived from the (derivative of) the mapping function $f$ in (1), and are not reported here for brevity (see [24] for details). It can be observed that, letting to zero the small parameters $\Delta_{2}$ and $\Delta_{3}$ [which parameterize the vanishing behavior of the coordinate transformation in (1)], the outermost layer $R_{3}<r<R_{4}$ reduces to the standard invisibility cloak [9] while the internal layer $R_{1}<r<R_{2}$ reduces to an anti-cloak [23] (with constitutive parameters opposite in sign to those of the inner target). It was shown in [24] that the two 
competing cloak/anti-cloak effects can be tailored so as to create an effectively cloaked region in the vacuum gap $R_{2}<r<R_{3}$, while still being able to restore a non-negligible field in the (lossless) inner cylinder. Such restored field may be interestingly used for sensing purposes, suggesting a novel route towards the sensor-cloaking idea. Therefore, it is of interest to study the scattering and absorption properties of the above configuration in the presence of a slightly lossy target in the inner region $\left(r<R_{1}\right)$, and explore the role of the various parameters available.

\section{Analytical results}

\section{A. General solution}

Assuming time-harmonic $[\exp (-i \omega t)]$ transverse-magnetic (TM) plane-wave excitation (with unit-amplitude $z$-directed magnetic field) impinging from the positive $x$-direction, the relevant magnetic field in the four-layer configuration of Fig. 1 can be computed via our previously derived Fourier-Bessel expansion [24], based on the approaches originally proposed in $[29,30,35,36]$,

$$
\begin{aligned}
H_{z}(r, \phi) & =\sum_{n=-\infty}^{\infty}\left\{\left(a_{n}^{(\nu)}+\delta_{\nu 5} i^{n}\right) J_{n}[g(r)]+b_{n}^{(\nu)} Y_{n}[g(r)]\right\} \\
& \times \exp (i n \phi), \quad R_{\nu-1}<r<R_{\nu}, \quad \nu=1, . ., 5 .
\end{aligned}
$$

In (2), $J_{n}$ and $Y_{n}$ denote the $n$ th-order Bessel functions of the first and second kind [37], respectively, $R_{0}=0$ and $R_{5}=\infty$ are "dummy" parameters introduced for notational convenience, $\delta_{p q}$ is the Kronecker delta, and

$$
g(r)=\left\{\begin{array}{l}
k_{0} r, \quad R_{2}<r<R_{3}, \\
\omega \sqrt{\epsilon^{\prime}[f(r)] \mu^{\prime}[f(r)]} f(r), \quad r<R_{2}, r>R_{3} .
\end{array}\right.
$$

Referring the reader to [24] for details on the procedure for computing the expansion coefficients $a_{n}^{(\nu)}$ and $b_{n}^{(\nu)}$, we limit ourselves to summarize the final results that are instrumental for what follows:

$$
\begin{gathered}
b_{n}^{(4,5)}=i a_{n}^{(5)}, \quad a_{n}^{(4)}=i^{n}+i a_{n}^{(5)}, \quad b_{n}^{(1,2)}=0, \quad a_{n}^{(2)}=a_{n}^{(1)}, \\
a_{0}^{(1,3,5)} \sim b_{0}^{(3)} \sim O\left(\frac{1}{\log \Delta_{3}}\right), \\
a_{n}^{(1)} \sim O\left(\frac{\Delta_{3}^{|n|}}{\Delta_{2}^{|n|}}\right), \quad a_{n}^{(3)} \sim b_{n}^{(3)} \sim O\left(\Delta_{3}^{|n|}\right), \quad a_{n}^{(5)} \sim O\left(\Delta_{3}^{2|n|}\right), \quad n>0,
\end{gathered}
$$


where the Landau asymptotic notation $O(\cdot)$ is used, neglecting irrelevant constants and higher-order terms. From (4)-(6), it is clear that, letting to zero $\Delta_{2}$ and $\Delta_{3}$ while maintaining their ratio finite, it is possible to render all the expansion coefficients pertaining to the vacuum gap and exterior region vanishingly small, while keeping the (higher-order) coefficients pertaining to the target region nonzero.

\section{B. Slightly mismatched target}

In the new sensor-cloaking scenario of interest, the inner region $r<R_{1}$ is filled by an absorbing particle (modeling a sensor or a detector), and thus, different from [24], is not matched with the surrounding anti-cloak. We therefore begin by studying perturbatively the effects of a slightly mismatched electrical permittivity,

$$
\bar{\varepsilon}_{1}=\left(1+\Delta_{\varepsilon}\right)^{2} \varepsilon_{1}
$$

of the target, with the vanishingly small term $\Delta_{\varepsilon}$ parameterizing this mismatch. Denoting with an overbar the expansion coefficients in (2) pertaining to the mismatched configuration, and repeating the procedure in [24], with the dependence on $\Delta_{\varepsilon}$ parameterized via a firstorder Taylor expansion, we obtain:

$$
\begin{gathered}
\bar{b}_{n}^{(1)}=0, \quad \bar{b}_{n}^{(4,5)}=i \bar{a}_{n}^{(5)}, \quad \bar{a}_{n}^{(4)}=i^{n}+\bar{a}_{n}^{(5)}, \\
\bar{b}_{n}^{(2)} \sim \bar{a}_{n}^{(1)} O\left(\Delta_{\varepsilon}\right), \quad \bar{a}_{n}^{(2)} \sim \bar{a}_{n}^{(1)}\left[1+O\left(\Delta_{\varepsilon}\right)\right], \\
\bar{a}_{n}^{(1)} \sim \frac{a_{n}^{(1)}}{1+O_{n}}, \quad\left\{\begin{array}{l}
\bar{a}_{n}^{(3,5)} \\
\bar{b}_{n}^{(3)}
\end{array}\right\} \sim\left\{\begin{array}{l}
a_{n}^{(3,5)} \\
b_{n}^{(3)}
\end{array}\right\}\left(\frac{1+O_{n}}{1+O_{n}}\right),
\end{gathered}
$$

where

$$
O_{n}= \begin{cases}O\left(\Delta_{\varepsilon} \log \Delta_{3}\right), & n=0 \\ O\left(\Delta_{\varepsilon} \Delta_{3}^{-2|n|}\right), & n>0 .\end{cases}
$$

In (9) and (10), for a better understanding of the scaling behaviors, the original (i.e., in the absence of mismatch) expansion coefficients have been factored out. It is readily observed that the mismatch affects primarily the field transmitted in the target region, while the field in the vacuum gap and the one scattered in the exterior space maintain the vanishingly small character as in the matched case. In particular, depending on ratio of the vanishingly small parameters $\Delta_{\varepsilon}$ and $\Delta_{3}$, the higher-order $\bar{a}_{n}^{(1)}$ coefficients will exhibit an algebraically decaying behavior. For instance, assuming $\Delta_{\varepsilon} \sim O\left(\Delta_{3}^{2 q}\right)$ (with $q>0$ ) and $\Delta_{2} \sim O\left(\Delta_{3}\right)$, it results from (10) [with (6)]

$$
\bar{a}_{n}^{(1)} \sim O\left(\Delta_{3}^{2|n|-2 q}\right), \quad n>q
$$


To sum up, the presence of a slightly mismatched target does not affect the cloaking function, but it may affect the anti-cloaking capability of restoring a field in the target region since, besides the zeroth-order coefficient $a_{0}^{(1)}$ in (10) (which is still logarithmically vanishing as in [24]), an infinite number of higher-order terms are now generally lost [cf. (12)]. For increasing mismatch levels, the anti-cloaking effect is progressively destroyed, and the cloaking effect eventually prevails.

\section{C. Vanishing-gap limit}

We now move on to studying the effects of the vacuum gap $R_{2}<r<R_{3}$ which, in the new scenario of interest, is no longer functional to creating a cloaked region, but it constitutes an additional degree of freedom to tailor the cloak/anti-cloak interactions. While its general effects in the EM response are not easy to parameterize, it is particularly insightful to study the limiting case,

$$
R_{3}=\left(1+\Delta_{G}\right) R_{2}
$$

where the term $\Delta_{G}$ parameterizes the the vanishingly small gap. Once again, proceeding as in [24], and parameterizing as a first-order Taylor expansion the dependence on $\Delta_{G}$, we obtain for the relevant expansion coefficients in the target and exterior regions:

$$
\begin{aligned}
& a_{n}^{(1)} \sim\left\{\begin{array}{l}
\frac{1}{1+O\left(\Delta_{G} \log \Delta_{3}\right)}, \quad n=0, \\
\frac{2 i^{n} \gamma^{|n|} \varepsilon_{1}}{\left(\varepsilon_{1}+\varepsilon_{0}\right)}\left(\frac{\Delta_{3}}{\Delta_{2}}\right)^{|n|}+O\left(\Delta_{3}^{2|n|}\right)+O\left(\Delta_{G}\right), \quad n \neq 0,
\end{array}\right. \\
& a_{n}^{(5)} \sim \begin{cases}\frac{O\left(\Delta_{G} \log ^{-1} \Delta_{3}\right)}{O\left(\Delta_{G}\right)+O\left(\log ^{-1} \Delta_{3}\right)}, & n=0, \\
O\left(\Delta_{3}^{2|n|}\right)\left[1+O\left(\Delta_{G}\right)\right], & n \neq 0,\end{cases}
\end{aligned}
$$

where

$$
\gamma=\frac{\sqrt{\varepsilon_{0} \mu_{0}} R_{4}\left(R_{2}-R_{1}\right)}{\sqrt{\varepsilon_{1} \mu_{1}} R_{1}\left(R_{4}-R_{3}\right)} .
$$

We observe that, in the vanishing-gap limit, the scattered-field coefficients maintain their vanishingly small character [in fact, the zero-th order terms vanish now faster than logarithmically, cf. (15)]. In connection with the field transmitted in the target region [cf. (14)], we point out that, besides the $(n \neq 0)$ th-order terms $\left[\right.$ for $\Delta_{2} \sim O\left(\Delta_{3}\right)$ ], it is now possible to

recover also the zeroth-order term by letting $\Delta_{G} \rightarrow 0$ faster than $\log ^{-1} \Delta_{3}$. In fact, assuming $\Delta_{G}=0$ (i.e., $R_{3}=R_{2}$ ), and enforcing the continuity of the coordinate transformation in (1), $f\left(R_{2}^{-}=R_{2}^{+}\right)$, we obtain

$$
\frac{R_{1} \Delta_{2}}{R_{2}+\Delta_{2}-R_{1}}=\frac{R_{4} \Delta_{3}}{R_{4}+\Delta_{3}-R_{3}}
$$


which, for $\Delta_{2,3} \rightarrow 0$, implies

$$
\gamma=\frac{\sqrt{\varepsilon_{0} \mu_{0}}}{\sqrt{\varepsilon_{1} \mu_{1}}}\left(\frac{\Delta_{2}}{\Delta_{3}}\right)
$$

For the (trivial) case of a vacuum target $\left(\varepsilon_{1}=\varepsilon_{0}, \mu_{1}=\mu_{0}\right)$, this yields $a_{n}^{(1)}=i^{n}$ in (14), i.e., the anti-cloak perfectly compensates the cloak, restoring the impinging plane-wave, as in the original scenario presented in [23]. For materials other than vacuum, the field transmitted in the target is a distorted version of the incident one.

\section{D. Remarks}

The two cases above (slightly mismatched target and vanishing gap) can readily be combined by substituting (14) and (15) in (10). From the above results, in the sensor-cloaking scenario of interest, the vanishing-gap configuration appears particularly well suited, since it allows the recovery of the (otherwise logarithmically vanishing) zeroth-order terms of the transmitted field (and thus, in principle, a more effective power absorption), while keeping the scattered field vanishingly small (with a faster decay of the zeroth-order terms). These considerations are expected to hold also in the case of slightly lossy cloak/anti-cloak shells.

\section{Parametric analysis}

\section{A. Generalities and observables}

To complement the analytical results above, we carried out a series of parametric studies, aimed at identifying the "optimal" parameter configurations and possible tradeoffs, within and beyond the limits $\Delta_{2,3, G, \varepsilon} \rightarrow 0$, and also in the more realistic case of slightly lossy cloak and anti-cloak.

In our studies below, we utilize the analytical solution in (2), and compactly parameterize the EM response of the configurations in terms of the total scattering and absorption crosssectional widths per unit length [33] (normalized to the vacuum wavelength $\lambda_{0}$ ),

$$
Q_{s}=\frac{2}{\pi} \sum_{n=-\infty}^{\infty}\left|\bar{a}_{n}^{(5)}\right|^{2}, \quad Q_{a}=-\frac{2}{\pi} \sum_{n=-\infty}^{\infty}\left\{\left|\bar{a}_{n}^{(5)}\right|^{2}+\operatorname{Re}\left[i^{-n} \bar{a}_{n}^{(5)}\right]\right\} .
$$

In the case of slightly lossy cloak and anti-cloak, for which the absorption cross-section $Q_{a}$ is no longer representative of the power absorption of the target/sensor only, we consider the time-averaged power (per unit length along the $z$-axis) dissipated in the target region,

$$
P_{a}=\frac{\omega \operatorname{Im}\left[\bar{\varepsilon}_{1}\right]}{2} \int_{0}^{R_{1}} r d r \int_{0}^{2 \pi} d \phi|\mathbf{E}(r, \phi)|^{2},
$$

with $\mathbf{E}$ denoting the local electric field.

The configuration under study is characterized by several geometrical and constitutive parameters, which render an exhaustive optimization computationally prohibitive. The designs 
presented below have been obtained by tweaking a limited number of parameters, until reaching reasonably good performance. Further improvements may be sought, although beyond the interest of the present paper.

\section{B. Representative results}

We begin considering the ideal case of lossless cloak and anti-cloak. For a lossy, small dielectric target/sensor with radius $R_{1}=\lambda_{0} / 8$ and $\varepsilon_{1}=(4+0.25 i) \varepsilon_{0}$, the plots in Fig. 2 illustrate the behavior of the observables of interest, as a function of $\Delta_{2}$ and $\Delta_{3}$, for $R_{2}=\lambda_{0} / 2$, $R_{3}=\left(1+\Delta_{G}\right) R_{2}, R_{4}=\lambda_{0}$, and three representative values of the vacuum gap thickness $\Delta_{G}$ in (13). More specifically, Figs. 2(a)-2(c) and 2(d)-2(f) show (in dB scale) the scattering and absorption cross-sectional widths (19), respectively, normalized to their values in vacuum (i.e., in the absence of the cloak/anti-cloak shells), viz.,

$$
\bar{Q}_{s} \equiv \frac{Q_{s}}{Q_{s}^{(0)}}, \quad \bar{Q}_{a} \equiv \frac{Q_{a}}{Q_{a}^{(0)}} .
$$

From the scattering responses [Figs. 2(a)-2(c)], we observe the presence of a broad minimum, whose position depends on the gap thickness $\Delta_{G}$. The case in the absence of gap [Fig. 2(a)] turns out to provide the most substantial scattering reduction (up to over $30 \mathrm{~dB}$ below the reference value in vacuum); such minimum is obtained for the smallest values of $\Delta_{2}$ and $\Delta_{3}$ in the observation range. Much weaker reductions, and different positions of the minima, are observed in the presence of even a small gap [Figs. 2(b) and 2(c)]. In all three cases, enhancements of the scattering responses (as compared with the reference values in vacuum) can be observed in certain regions; such superscattering phenomena [25] are typical of complementary media, but are not of interest in the present investigation.

The absorption responses [Figs. 2(d)-2(f)] exhibit a slightly more complex structure, with the presence of local minima and maxima, qualitatively similar for the different gap thicknesses. Also in these cases, some superabsorption phenomena [26] are visible, but they are generally associated with the above superscattering effects, and therefore not functional in the sensor-cloaking scenario of interest.

In agreement with the predictions from the analytical study in Sec. 3, the potentially more interesting responses (especially in terms of scattering reductions) are obtained in the absence of the gap. However, it can be observed that the maximum scattering reduction generally does not coincide with the maximum absorption, and different parameters choices (e.g., increasing the normalized absorption at the expense of the scattering reduction, and viceversa) are possible, as summarized in the tradeoff curve (full square markers) in Fig. 3. Such curve, extracted from Figs. 2(a) and 2(d), for a given value of the (normalized) scattering response yields the largest (normalized) absorption response attainable. In particular, it illustrates how, in the absence of the gap and varying $\Delta_{2}$ and $\Delta_{3}$, it is possible to span the entire 
range of cloak/anti-cloak interactions, going from a regime featuring weak scattering and weak absorption (i.e., cloak-prevailing) to one featuring scattering and absorption levels comparable with those in vacuum (i.e., anti-cloak-prevailing). However, the slope of the curve is such that, in an intermediate regime, one can attain significant scattering reductions (e.g., -20 dB) while maintaining sensible absorption levels (e.g., -1.56dB). Figure 4 shows a representative field map, from which the very weak overall scattering, and yet the powercoupling capabilities, are fairly visible; as a reference, the case of the target/sensor freestanding in vacuum is shown in Fig. 5.

For comparison purposes, Fig. 3 also shows (full markers) the tradeoff curves pertaining to different levels of target losses $\left(\operatorname{Im}\left[\bar{\varepsilon}_{1}\right]\right)$, from which one notes that loss (and hence mismatch) increases result in a performance degradation (i.e., lower normalized absorption for a given scattering reduction), in accord with our theoretical predictions in Sec. 3.

One might argue that a similar trend (i.e., scattering reduction at the expenses of absorption) is also qualitatively exhibited by an imperfect cloak alone, and might therefore wonder to what extent the anti-cloak is strictly necessary and functional. In order to address this issue, Fig. 3 also shows (empty markers) the reference curves pertaining to a TO-based imperfect cloak characterized by the standard (linear) coordinate transformation

$$
r^{\prime}=f(r)=\left\{\begin{array}{l}
r, \quad r<R_{1}, \quad r>R_{4}, \\
R_{4}\left(\frac{r-R_{1}+\Delta_{1}}{R_{4}-R_{1}+\Delta_{1}}\right), \quad R_{1}<r<R_{4},
\end{array}\right.
$$

and the same total thickness $\left(R_{4}-R_{1}\right)$ as the above cloak/anti-cloak configuration. Note that, in this case, the resulting tradeoff curves are obtained by simply scanning the only parameter $\left(\Delta_{1}\right)$ available. One can observe that, in spite of the anticipated qualitatively similar trends, the tradeoff curves pertaining to the imperfect cloak alone are considerably steeper, and therefore yield acceptable scattering reductions only at the expenses of comparable reductions in the absorption.

We also considered more realistic cases involving lossy cloak and anti-cloak shells. More specifically, for the (DPS) cloak shell, we assumed a loss-tangent of 0.001, while for the (DNG) anti-cloak we considered a higher value (0.01). The corresponding scattering and absorption normalized parameters are shown in Fig. 6, with the time-averaged dissipative power in (20) replacing the absorption cross-sectional width in (19). As compared with the lossless case above, qualitatively similar results are observed, with an expectable loss-induced moderate deterioration in the performance. Again, the case in the absence of the gap [Figs. 6(a) and $6(d)$ ] turns out to provide the more interesting responses. Figure 7 shows the corresponding tradeoff curves, from which, by comparison with the lossless case in Fig. 3, one observes a reduced dynamics in the scattering reduction and a slight increase in the slope. Figure 8 
shows a reference field map, for a configuration featuring a scattering reduction of $20 \mathrm{~dB}$ at the expense of an absorption reduction of $\sim 3 \mathrm{~dB}$, which qualitatively resembles the lossless case above.

Finally, Fig. 9 shows the effects in the tradeoff curves of a further increase of the losstangent (0.1) in the (DNG) anti-cloak. Again, further compression in the $\bar{Q}_{s}$ dynamics and increase in the slope are observed. Nevertheless, in spite of the relatively high loss level, in the best-performing case $\left(\operatorname{Im}\left[\bar{\varepsilon}_{1}\right]=0.25 \varepsilon_{0}\right)$, one can still achieve a scattering reduction of nearly $15 \mathrm{~dB}$ at the expense of a reduction of $\sim 4 \mathrm{~dB}$ in the absorption. The corresponding field map is shown in Fig. 10.

\section{Implementation issues}

Although the present prototype study is mainly aimed at providing a proof of principle of the cloak/anti-cloak-based sensor-invisibility effect, it is still important to address the related implementation issues. In particular, for the proposed configuration, the major technological challenges associated with the practical realization of the involved transformation media are related to the spatial inhomogeneity (with extreme values) of all constitutive-parameter components, their general magnetic character, and, most of all, the DNG character of the anti-cloak (in view of the assumed DPS target).

The first two issues are inherent of TO-based cloaks, and can be addressed via rigorous or approximate approaches. In particular, higher-order coordinate transformations have been proposed in order to obtain a perfect cloak with spatially invariant axial material parameters [38]. Non-magnetic implementations have also been proposed, based on approximate reductions of the constitutive parameters that preserve the ray trajectories inside the cloak shell (see, e.g., [39-42]) as well as on mapping from a nearly transparent, anisotropic and spatially inhomogeneous fictitious domain [43].

On the other hand, the last issue (DNG character) is specifically tied with the anti-cloak concept [23], although it may be transferred, in principle, to the target [24]. Nevertheless, we have shown [44] in some preliminary studies on square-type configurations (similar to that originally proposed in [45]) the intriguing possibility of achieving approximate anti-cloaking

effects using DPS media only. Extension of these preliminary results to the sensor-cloaking scenario is therefore a key issue, and is currently being pursued.

To sum up, a candidate practical implementation may be envisioned as based on the above approximate DPS anti-cloak in conjunction with a suitable parameter reduction approach [38-43]. The study of such a configuration, which would generally require non-trivial modifications in our analytical framework or a fully-numerical approach, is beyond the scope of the present prototype study, and will be the subject of forthcoming publications. 


\section{Conclusions and perspectives}

In this paper, we have reported our study of cloak/anti-cloak interactions in 2-D cylindrical geometries, aimed at exploring the potential of such mechanisms in a sensor-cloaking scenario. Via analytical and parametric studies, we have shown that it is possible to tailor the cloak/anti-cloak interactions so as to strongly reduce the scattering response of a slightly lossy cylindrical target (mimicking a "sensor") while still allowing effective dissipative power coupling, with a controllable tradeoff. Such effects are not destroyed by the presence of slight/moderate losses in the cloak/anti-cloak.

These results lay the foundations for a possible TO-based route to sensor-cloaking, alternative to the insofar proposed approaches [32-34]. Within this framework, a comparative study of the various approaches is in order. For instance, one difference that may be expected between our approach (which generally requires DNG resonant metamaterials) and the scattering-cancellation approach in [32] (which is based on inherently penetrable and non-resonant materials) is the presence in our case of a "delay" effect (typical of DNG-based devices) due to the buildup-time of the resonant field in the anti-cloak.

Current and future studies are also aimed at removing or mitigating the major technological challenges associated with the practical realization of the involved transformation media (see Sec. 5), as well as at the extension to the full 3-D (vector) scenario.

\section{Acknowledgments}

A. Alù was partially supported by the National Science Foundation (NSF) CAREER Grant No. ECCS-0953311.

\section{References}

1. A. Alù and N. Engheta, "Achieving transparency with plasmonic and metamaterial coatings," Phys. Rev. E 72, 016623 (2005).

2. M. G. Silveirinha, A. Alù, and N Engheta, "Cloaking mechanism with antiphase plasmonic satellites," Phys. Rev. B 78, 205109 (2008).

3. G. W. Milton and N. A. P. Nicorovici, "On the cloaking effects associated with anomalous localized resonance," Proc. R. Soc. London A 462, 3027-3059 (2006).

4. U. Leonhardt, "Optical conformal mapping," Science 312, 1777-1780 (2006).

5. J. B. Pendry, D. Schurig, and D. R. Smith, "Controlling electromagnetic fields," Science 312, 1780-1782 (2006).

6. A. Håkansson, "Cloaking of objects from electromagnetic fields by inverse design of scattering optical elements," Opt. Express 15, 4328-4334 (2007). 
7. P. Alitalo, O. Luukkonen, L. Jylha, J. Venermo, and S. A. Tretyakov, "Transmission-line networks cloaking objects from electromagnetic fields," IEEE Trans. Antennas Propag. 56, 416-424 (2008).

8. I. I. Smolyaninov, V. N. Smolyaninova, A. V. Kildishev, and V. M. Shalaev, "Anisotropic metamaterials emulated by tapered waveguides: Application to optical cloaking," Phys. Rev. Lett. 102, 213901 (2009).

9. D. Schurig, J. J. Mock, B. J. Justice, S. A. Cummer, J. B. Pendry, A. F. Starr, and D. R. Smith, "Metamaterial electromagnetic cloak at microwave frequencies," Science 314, 977-980 (2006).

10. I. I. Smolyaninov, Y. J. Hung, and C. C. Davis, "Two-dimensional metamaterial structure exhibiting reduced visibility at $500 \mathrm{~nm}, "$ Opt. Lett. 33, 1342-1344 (2008).

11. S. Tretyakov, P. Alitalo, O. Luukkonen, and C. Simovski, "Broadband electromagnetic cloaking of long cylindrical objects," Phys. Rev. Lett. 103, 103905 (2009).

12. B. Edwards, A. Alù, M. G. Silveirinha, and N. Engheta, "Experimental verification of plasmonic cloaking at microwave frequencies," Phys. Rev. Lett. 103, 153901 (2009).

13. V. M. Shalaev, "Transforming light," Science 322, 384-386 (2008).

14. U. Leonhardt and T. G. Philbin, "Transformation optics and the geometry of light," Prog. Opt. 53, 69-152 (2009).

15. J. Li and J. B. Pendry, "Hiding under the carpet: A new strategy for cloaking," Phys. Rev. Lett. 101, 203901 (2008).

16. R. Liu, C. Ji, J. J. Mock, J. Y. Chin, T. J. Cui, and D. R. Smith, "Broadband groundplane cloak," Science 323, 366-369 (2009).

17. L. H. Gabrielli, J. Cardenas, C. B. Poitras, and M. Lipson, "Silicon nanostructure cloak operating at optical frequencies," Nature Photon. 3, 461-463 (2009).

18. J. Valentine, J. Li, T. Zentgraf, G. Bartal, and X. Zhang, "An optical cloak made of dielectrics," Nature Mater. 8, 568-571 (2009).

19. T. Ergin, N. Stenger, P. Brenner, J. B. Pendry, and M. Wegener, "Three-dimensional invisibility cloak at optical wavelengths," Science 328, 337-339 (2010).

20. H. F. Ma and T. J. Cui, "Three-dimensional broadband ground-plane cloak made of metamaterials, Nature Commun. 1, 1-6 (2010).

21. H. Ma, S. Qu, Z. Xu, and J. Wang, "The open cloak," Appl. Phys. Lett. 94, 103501 (2009).

22. Y. Lai, H. Chen, Z.-Q. Zhang, and C. T. Chan, "Complementary media invisibility cloak that cloaks objects at a distance outside the cloaking shell," Phys. Rev. Lett. 102, 093901 (2009).

23. H. Chen, X. Luo, H. Ma, and C. T. Chan, "The anti-cloak," Opt. Express 16, 1460314608 (2008). 
24. G. Castaldi, I. Gallina, V. Galdi, A. Alù, and N. Engheta, "Cloak/anti-cloak interactions," Opt. Express 17, 3101-3114 (2009).

25. T. Yang, H. Chen, X. Luo, and H. Ma, "Superscatterer: Enhancement of scattering with complementary media," Opt. Express 16, 18545 (2008).

26. J. Ng, H. Chen, and C. T. Chan, "Metamaterial frequency-selective superabsorber," Opt. Lett. 34, 644-464 (2009).

27. H. Chen, C. T. Chan, S. Liu, and Z. Lin, "A simple route to a tunable electromagnetic gateway," New J. Phys. 11, 083012 (2009).

28. Y. Lai, J. Ng, H. Chen, D. Han, J. Xiao, Z.-Q. Zhang, and C. T. Chan, "Illusion optics: The optical transformation of an object into another object," Phys. Rev. Lett. 102, 253902 (2009).

29. Z. Ruan, M. Yan, C. W. Neff, and M. Qiu, "Ideal cylindrical cloak: Perfect but sensitive to tiny perturbations," Phys. Rev. Lett. 99, 113903 (2007).

30. B. Zhang, H. S. Chen, B. I. Wu, Y. Luo, L. X. Ran, and J. A. Kong, "Response of a cylindrical invisibility cloak to electromagnetic waves," Phys. Rev. B 76, 121101 (2007).

31. B. Zhang, H. Chen, B. I. Wu, and J. A. Kong, "Extraordinary surface voltage effect in the invisibility cloak with an active device inside," Phys. Rev. Lett. 100, 063904 (2008).

32. A. Alù and N. Engheta, "Cloaking a sensor," Phys. Rev. Lett. 102, 233901 (2009).

33. Z. Ruan and S. Fan, "Temporal coupled-mode theory for Fano resonance in light scattering by a single obstacle," J. Phys. Chem. C 114, 7324-7329 (2010).

34. A. Greenleaf, Y. Kurylev, M. Lassas, and G. Uhlmann, "Cloaking vs. shielding in transformation optics," preprint arXiv:0912.1872v1 [physics.optics] (2009).

35. H. S. Chen, B. I. Wu, B. Zhang, and J. A. Kong, "Electromagnetic wave interactions with a metamaterial cloak," Phys. Rev. Lett. 99, 063903 (2007).

36. Y. Luo, H. Chen, J. Zhang, L. Ran, and J. A. Kong, "Design and analytical full-wave validation of the invisibility cloaks, concentrators, and field rotators created with a general class of transformations," Phys. Rev. B 77, 125127 (2008).

37. M. Abramowitz and I. A. Stegun, Handbook of Mathematical Functions, Ninth printing (Dover, New York, 1970).

38. Y. Luo, J. Zhang, H. Chen, S. Xi, and B.-I. Wu, "Cylindrical cloak with axial permittivity/permeability spatially invariant," Appl. Phys. Lett. 93, 033504 (2008).

39. S. A. Cummer, B.-I. Popa, D. Schurig, D. R. Smith, and J. Pendry, "Full-wave simulations of electromagnetic cloaking structures," Phys. Rev. E 74, 036621 (2006).

40. W. Cai, U. K. Chettiar, A. V. Kildishev, V. M. Shalaev, and G. W. Milton, "Nonmagnetic cloak with minimized scattering," Appl. Phys. Lett. 91, 111105 (2007).

41. I. Gallina, G. Castaldi, and V. Galdi, "A higher-order optical transformation for nonmagnetic cloaking," Microwave Opt. Technol. Lett. 50, 3186 (2008). 
42. L. Zhang, M. Yan, and M. Qiu, "The effect of transformation order on the invisibility performance of a practical cylindrical cloak," J. Opt. A Pure Appl. Opt. 10, 095001 (2008).

43. G. Castaldi, I. Gallina, and V. Galdi, "Nearly perfect nonmagnetic invisibility cloaking: Analytic solutions and parametric studies," Phys. Rev. B 80, 125116 (2009).

44. I. Gallina, G. Castaldi, V. Galdi, A. Alù, and N. Engheta, "General class of metamaterial transformation slabs," Phys. Rev. B 81, 125124 (2010).

45. M. Rahm, D. Schurig, D. A. Roberts, S. A. Cummer, D. R. Smith, and J. B. Pendry, "Design of electromagnetic cloaks and concentrators using form-invariant coordinate transformations of Maxwells equations," Photon. Nanostruct. 6, 87-95 (2008). 


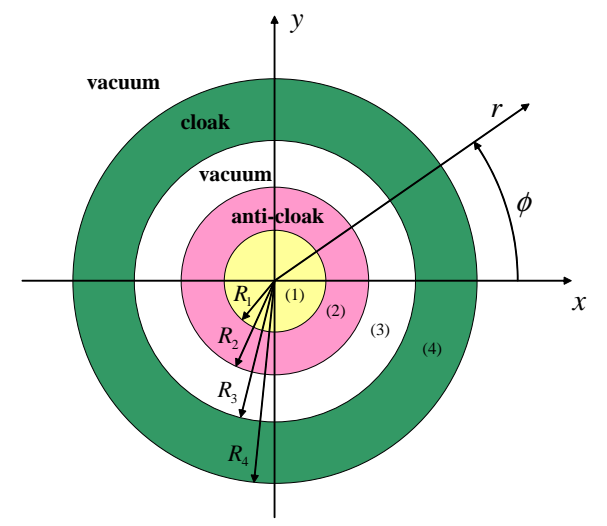

Fig. 1. (Color online) Problem geometry: A homogeneous metamaterial circular-cylindrical target of radius $R_{1}$ and constitutive parameters $\varepsilon_{1}$ and $\mu_{1}$, surrounded by a cloak and anti-cloak cylindrical shells separated by a vacuum gap, in the actual physical space $(x, y, z)$ [and associated cylindrical coordinate system $(r, \phi, z)]$. 

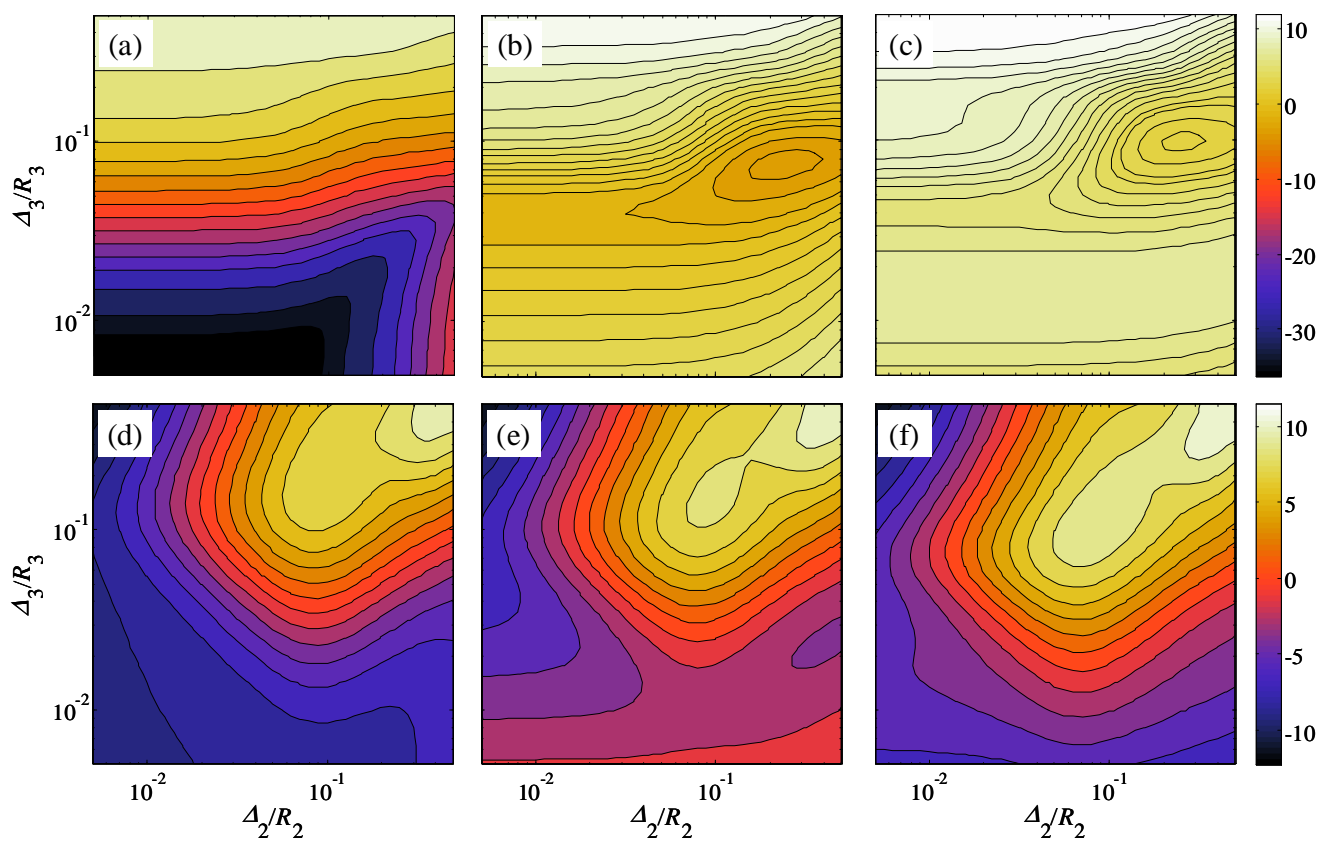

Fig. 2. (Color online) Geometry as in Fig. 1, with $\bar{\varepsilon}_{1}=(4+i 0.25) \varepsilon_{0}, \mu_{1}=\mu_{0}$, $R_{1}=\lambda_{0} / 8, R_{2}=\lambda_{0} / 2, R_{3}=\left(1+\Delta_{G}\right) R_{2}, R_{4}=\lambda_{0}$, and lossless cloak and anti-cloak. (a)-(c) Total scattering cross-sectional width in (19) normalized by the reference value in vacuum [cf. (21)] in $\mathrm{dB}$ scale, as a function of $\Delta_{2} / R_{2}$ and $\Delta_{3} / R_{3}$, for gap thickness $\Delta_{G}=0,1 / 50,1 / 25$, respectively. (d)-(f) Corresponding total absorption cross-sectional width normalized by the reference value in vacuum. 


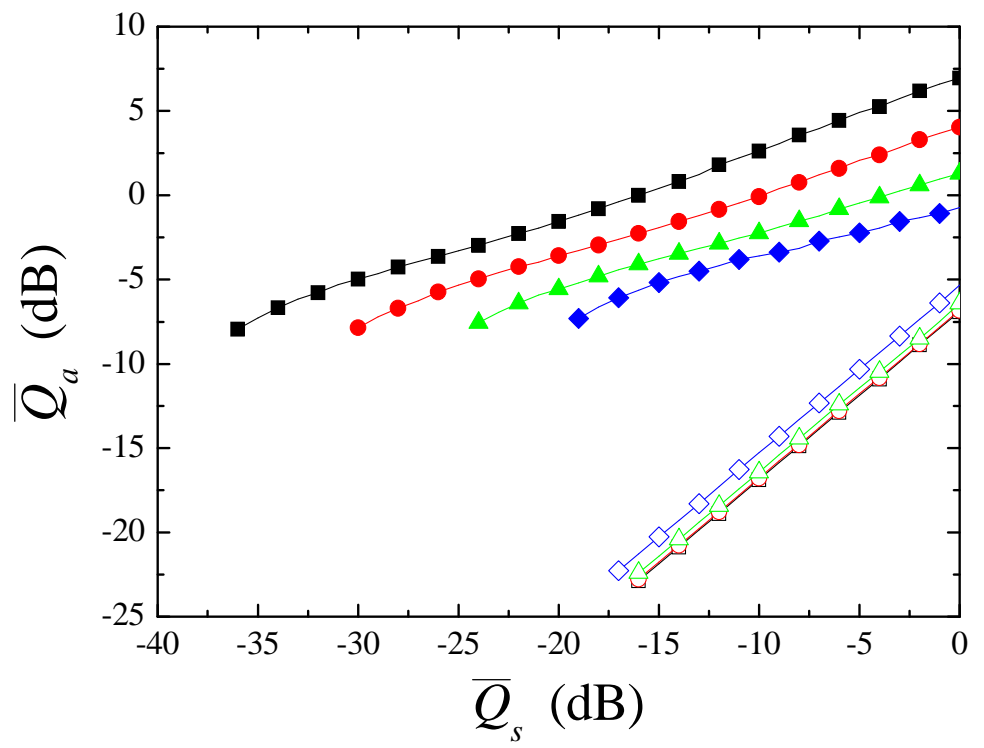

Fig. 3. (Color online) Tradeoff curves (full markers) pertaining to the lossless cloak/anti-cloak configuration in Fig. 2, for various values of the target loss levels (squares, circles, triangles, diamonds: $\operatorname{Im}\left[\bar{\varepsilon}_{1}\right]=0.25 \varepsilon_{0}, 0.5 \varepsilon_{0}, \varepsilon_{0}, 2 \varepsilon_{0}$, respectively). Also shown (empty markers), as references, are the corresponding curves pertaining to an imperfect (lossless) cloak configuration [cf. (22)]. 


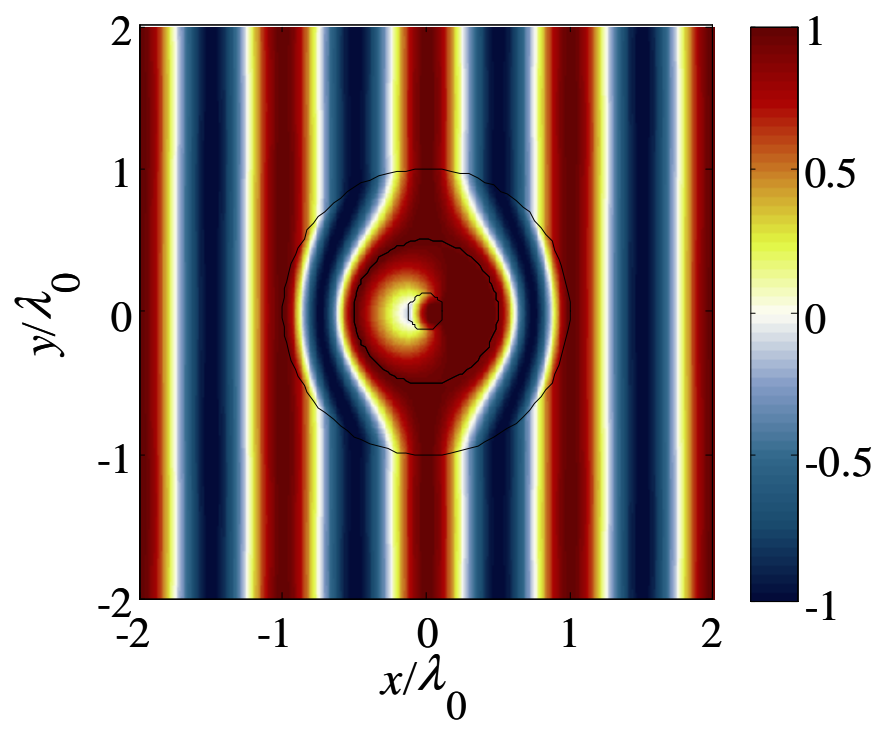

Fig. 4. (Color online) Geometry and parameters as in Fig. 2. Field map (real part of magnetic field) pertaining to a plane-wave-excited lossless cloak/anticloak configuration with $\Delta_{G}=0, \Delta_{2} / R_{2}=0.118, \Delta_{3} / R_{3}=0.03$, featuring $\bar{Q}_{s}=-20 \mathrm{~dB}$ and $\bar{Q}_{a}=-1.56 \mathrm{~dB}$.

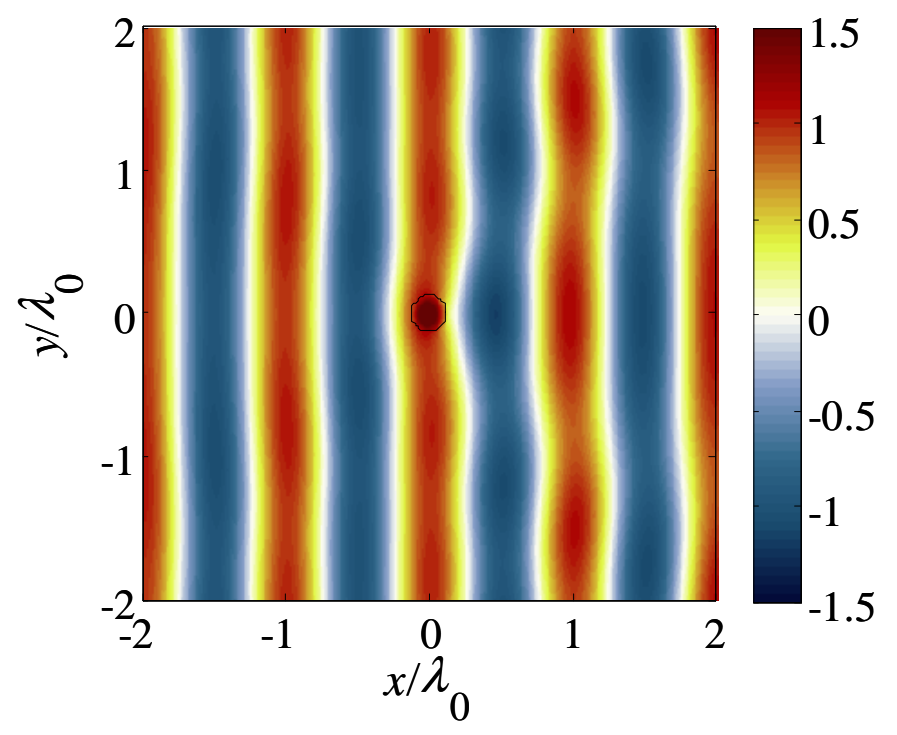

Fig. 5. (Color online) As in Fig. 4, but for the target free-standing in vacuum. 

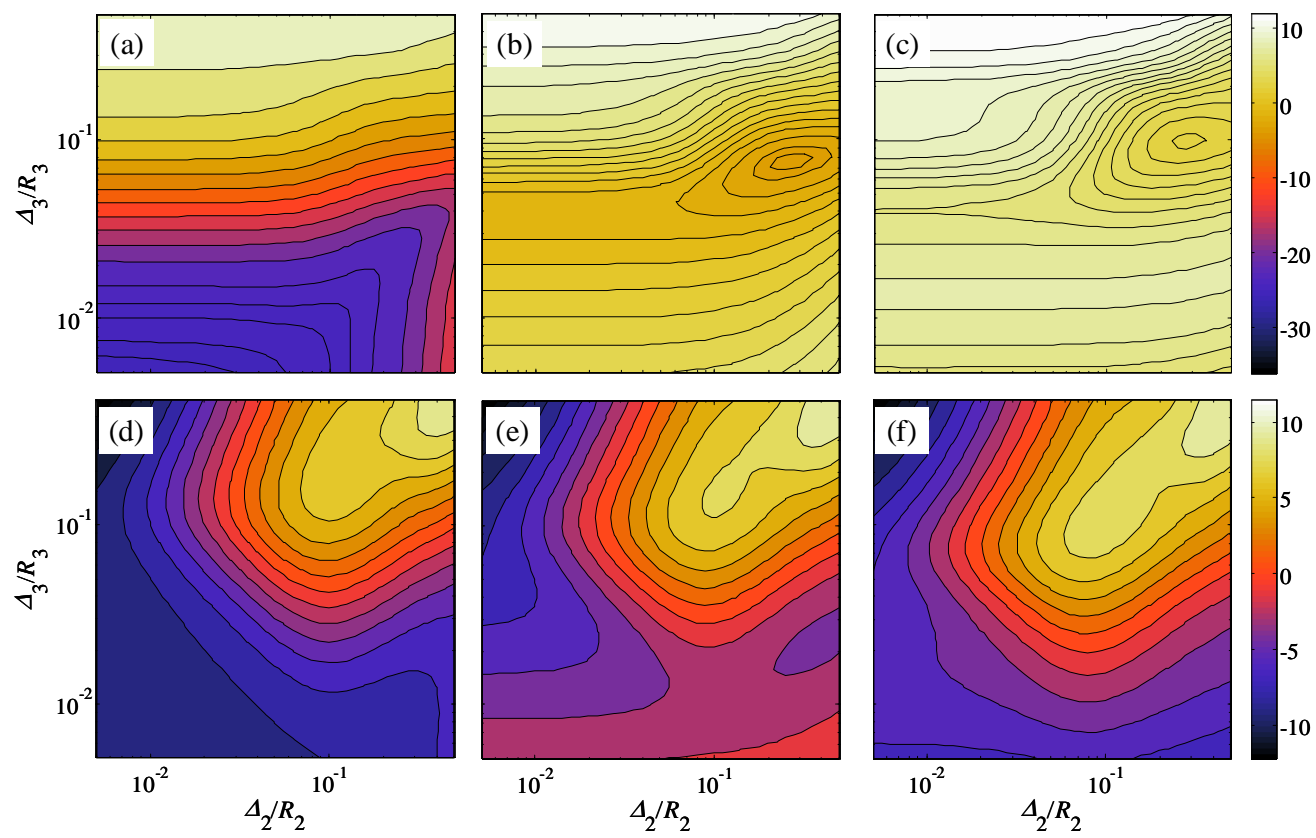

Fig. 6. (Color online) As in Fig. 2, but for a lossy cloak (loss-tangent=0.001) and anti-cloak (loss-tangent=0.01) configuration. In (d)-(f), the time-averaged dissipative power $P_{a}$ in (20) (normalized to its free space value $P_{a}^{(0)}$ ) is considered. 


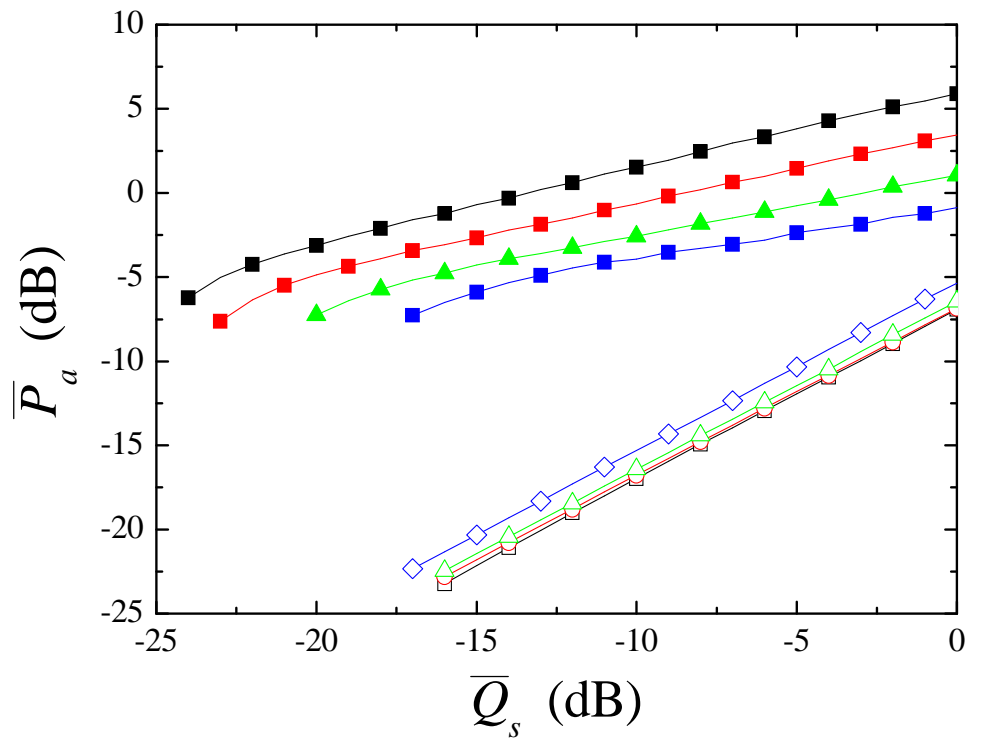

Fig. 7. (Color online) As in Fig. 3, but for a lossy cloak (loss-tangent=0.001) and anti-cloak (loss-tangent $=0.01$ ) configuration.

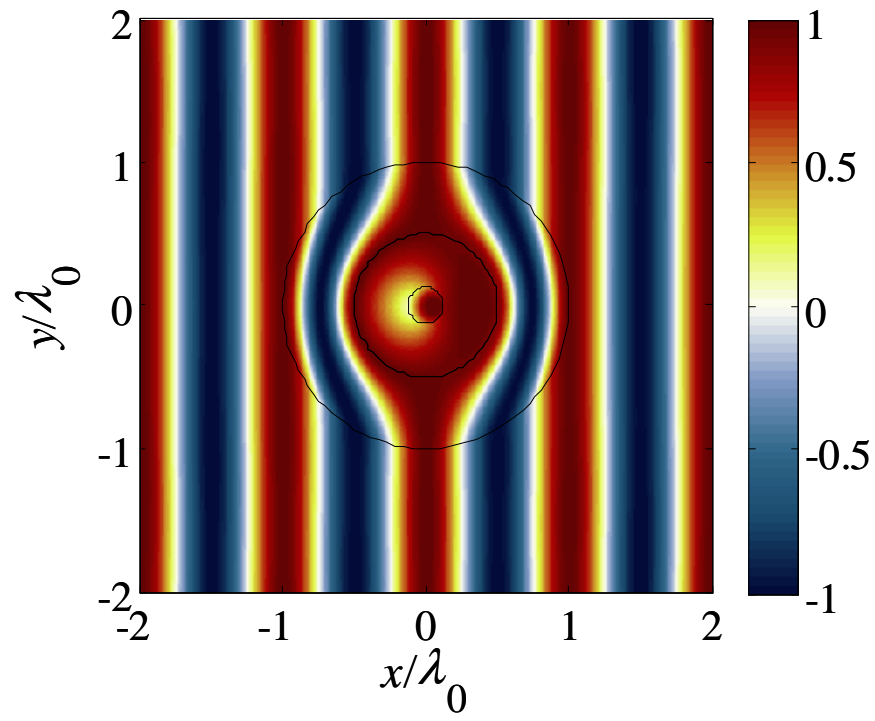

Fig. 8. (Color online) As in Fig. 4, but for a slightly lossy cloak (losstangent $=0.001$ ) and anti-cloak (loss-tangent $=0.01)$ configuration with $\Delta_{G}=0$, $\Delta_{2} / R_{2}=0.158, \Delta_{3} / R_{3}=0.028$, featuring $\bar{Q}_{s}=-20 \mathrm{~dB}$ and $\bar{P}_{a} \equiv P_{a} / P_{a}^{(0)}=$ $-3.13 \mathrm{~dB}$. 


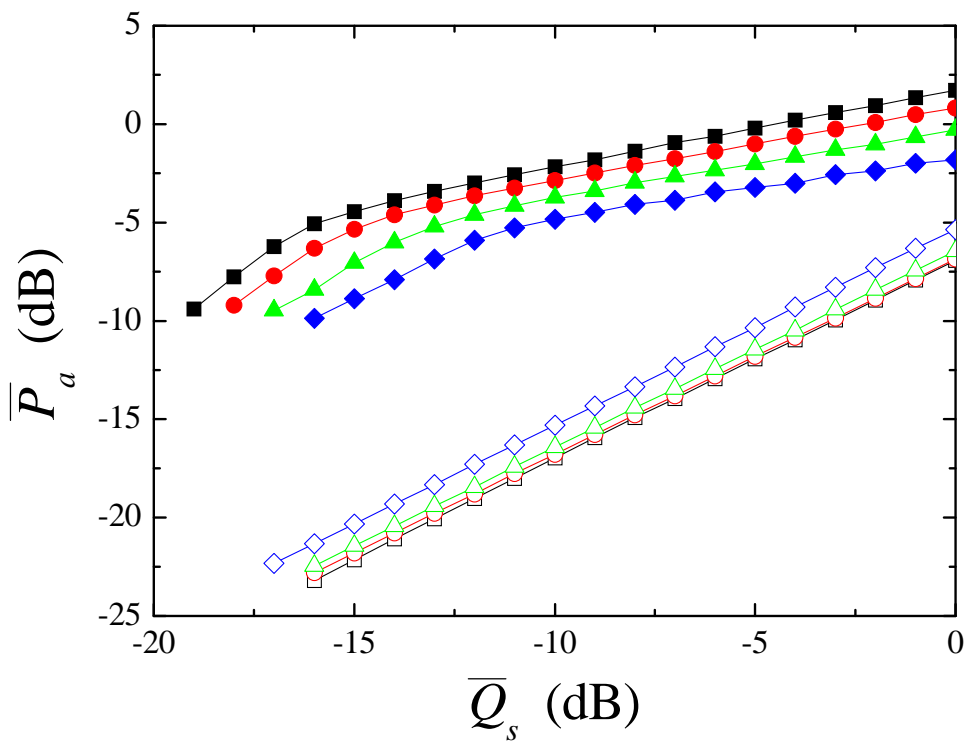

Fig. 9. (Color online) As in Fig. 3, but for increased loss level (losstangent $=0.1)$ in the anti-cloak.

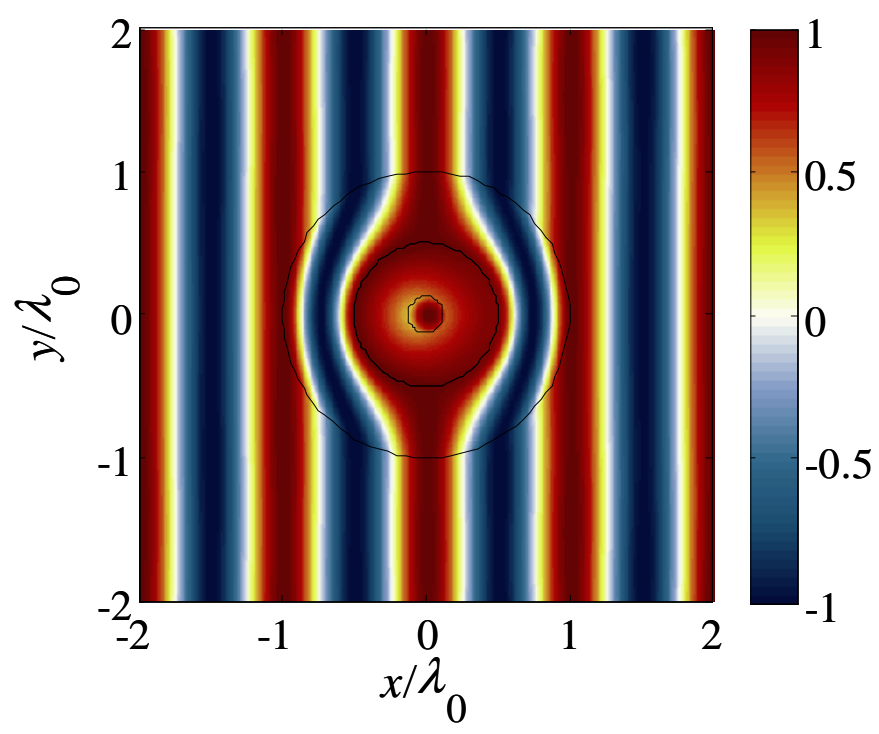

Fig. 10. (Color online) As in Fig. 4, but for a lossy cloak (loss-tangent=0.001) and anti-cloak (loss-tangent $=0.1$ ) configuration with $\Delta_{G}=0, \Delta_{2} / R_{2}=0.315$, $\Delta_{3} / R_{3}=0.037$, featuring $\bar{Q}_{s}=-15 \mathrm{~dB}$ and $\bar{P}_{a}=-4.44 \mathrm{~dB}$. 